\title{
Revisiting Smell and Taste Dysfunctions during COVID-19 Pandemic in Hong Kong
}

\author{
Ryan HW Cho ${ }^{1}$, Zion WH To ${ }^{1}$, Zenon WC Yeung ${ }^{1}$, Eugene YK Tso ${ }^{2}$, Kitty SC Fung ${ }^{3}$, Sandy KY Chau ${ }^{4}$, \\ Erica YL Leung ${ }^{5}$, Thomas SC Hui ${ }^{1}$, Steven WC Tsang ${ }^{5}$, KN Kung ${ }^{2}$, Eudora YD Chow ${ }^{3}$, Victor Abdullah ${ }^{1}$, \\ C Andrew van Hasselt ${ }^{6}$, Michael CF Tong ${ }^{6 *}$ and Peter KM Ku${ }^{1,6 *}$
}

${ }^{1}$ Department of Otorhinolaryngology, Head and Neck Surgery, United Christian Hospital and Tseung Kwan O Hospital, Hong Kong

${ }^{2}$ Department of Medicine and Geriatric, United Christian Hospital, Hong Kong

${ }^{3}$ Department of Pathology, United Christian Hospital, Hong Kong

${ }^{4}$ Department of Pathology, Tseung Kwan O Hospital, Hong Kong

${ }^{5}$ Department of Medicine, Tseung Kwan O Hospital, Hong Kong

${ }^{6}$ Department of Otorhinolaryngology, Head and Neck Surgery, United Christian Hospital and Tseung Kwan O Hospital, Hong Kong

*Corresponding author: Peter KM Ku, Department of Otorhinolaryngology, Head and Neck Surgery, United Christian Hospital and Tseung Kwan O Hospital, 2 Po Ning Lane, New Territories, Hong Kong

Contributed Equally: *Peter KM Ku and * Michael CF Tong

To Cite This Article: Peter KM Ku. Revisiting Smell and Taste Dysfunctions during COVID-19 Pandemic in Hong Kong. 2020 - 9(3). AJBSR. MS.ID.001394. DOI: 10.34297/AJBSR.2020.09.001394.

Received: 啙: June 11, 2020; Published: 策 June 30, 2020

\begin{abstract}
Background: This cross-sectional cohort study in Hong Kong aimed to revisit smell and taste symptoms in COVID-19 in Chinese population which were previously underreported in China.

Methods: 120 symptomatic patients underwent testing for SARS-coronavirus 2 (SARS-CoV-2) were recruited. The clinical data of confirmed COVID-19 ( $\mathrm{n}=60)$ and control subjects $(\mathrm{n}=60)$ were collected, including severity of smell and taste impairment and the cycle threshold (Ct) values from a SARS-CoV-2 polymerase chain reaction (PCR) assay of specimens.
\end{abstract}

Results: Twenty-eight (46.7\%) and twenty-seven (45\%) COVID-19 patients reported smell and taste impairment, respectively, whereas neither symptom occurred in the control group $(\mathrm{p}<0.05)$. Cough $(60 \%)$, fever $(50 \%)$, expectorant $(43 \%)$ and dyspnea $(25 \%)$ were common symptoms in COVID-19 but were not specific to the diagnosis.

Conclusion: We observed no ethnic differences in COVID-19-related smell and taste dysfunctions between Chinese and Caucasians. These symptoms were more specific for COVID-19 than fever, cough, expectorant and dyspnea

Keywords: Smell, Taste, COVID-19, Dysfunction

\section{Introduction}

The 2019 novel coronavirus disease (COVID-19) pandemic has led to the tragic loss of more than 300,000 lives worldwide [1], as well as a global economic recession largely triggered by nationwide lockdowns imposed by governments as attempted disease control measures. The disease is caused by the SARSCoV-2 virus and is considered a prodigious worldwide threat to human health. A joint press release from the British Rhinologic
Society and British Association of Otorhinolaryngology on March 21, 2020 reported that an increasing number of COVID-19 patients in Italy, South Korea, Iran, and United Kingdom had reported smell impairments [2]. Professional organizations in the United States and United Kingdom have worked quickly to produce public guidelines regarding smell and taste disturbances that individuals with COVID-19 may experience [3,4]. At the time of writing, there have been more reports and scientific publications on the smell 
and taste disturbance in COVID-19 to incidence, symptomatology, objective tests and possibly recovery in the West [5,6]. However, no case series has been reported to re-evaluate smell and taste impairment in Chinese patients with COVID-19 outside province of China, despite an apparently low incidence of these symptoms in previous large cohort studies [7-9].

Both the United States and European countries have reported a high incidence of smell (68-85.6\%) and taste dysfunction (71-88\%) among patients with COVID-19 [10,11]. In contrast, Korea reported that only $15.3 \%$ of a large cohort of patients $(\mathrm{N}=3,191)$ reported a loss of smell or taste [12]. It is therefore unclear whether these symptoms are affected by ethnic differences or other geographical factors. Here, we aim to describe our preliminary evaluation of smell and taste dysfunction associated with COVID-19 through a cross-sectional cohort study of an ethnic Chinese population in Hong Kong. Future publications from Western and Asian countries will provide a comparative comprehensive overview of these specific symptoms and their potential use as specific screening tools for COVID-19.

\section{Materials and Methods}

This study adhered to the tenets of the Declaration of Helsinki. The study protocol was approved by the Research Ethics Committee of Kowloon Central and Kowloon East Cluster of The Hospital Authority of Hong Kong. Informed consent was obtained from all patients.

\section{Study Design}

This is a cross-sectional cohort study. Data collection is ongoing from all patients with suspected and confirmed COVID-19 during this pandemic. For this preliminary analysis, we recruited subjects from 120 out of 165 patients who had been tested for SARScoronavirus 2 (SARS-CoV-2) and were admitted to the United Christian Hospital and Tseung Kwan 0 Hospital between February 8 and April 15, 2020. The inclusion criteria were: aged $\geq 18$ years; a SARS-CoV-2 test history; and an ability understand written traditional Chinese or verbal Cantonese or Mandarin. The exclusion

\section{Results}

criteria were: aged $<18$ years and having pre-existing smell and taste disturbances. The COVID-19 group comprised patients with a positive test result for SARS-CoV-2 from combined nasopharyngeal swab (NPS) and throat swab (TS). The control group comprised patients with suspected COVID-19 who had a negative SARS-CoV-2 test result.

\section{Questionnaire Design and Data Collection}

A traditional Chinese-language questionnaire was designed to collect the patients' clinical data via phone contact or online submission. Information was collected on history of rhinitis, nasal surgery, use of medication for nasal symptoms, and symptoms such as nasal discharge, nasal obstruction, purulent nasal discharge, blood-stained nasal discharge, cough, sputum, shortness of breath (SOB), fever, change of smell and change of taste. A 10-point scale was used to indicate changes in smell and taste (1: anosmia and ageusia, 2-9: hyposmia and hypogeusia, 10: no change).

The participants' demographic characteristics, travel history, occupation, cluster phenomenon, contact history and laboratory findings were extracted from electronic medical records. Of particular importance were the cycle threshold $(\mathrm{Ct})$ values from a SARS-CoV-2 polymerase chain reaction (PCR) assay of combined nasopharyngeal and throat swabs (NPS + TS).

\section{Statistical analysis}

All statistical analyses were performed using SPSS version 23.0 (IBM, Armonk, NY, USA). Symptom variables included in the comparisons were runny nose, nasal obstruction, blood-stained or purulent nasal discharge, cough, sputum, SOB, fever and change in smell or taste. A logistic regression analysis was used to compute the odds ratios (OR) of the symptoms. Student's t-test and Pearson's chi-square test were used to compare demographic statistics and questionnaire results between groups. The Mann-Whitney U test was used to compare smell and taste impairments between groups. The relationship between smell and taste impairments was analyzed using Spearman's rank correlation coefficient. A p value < 0.05 was considered statistically significant.

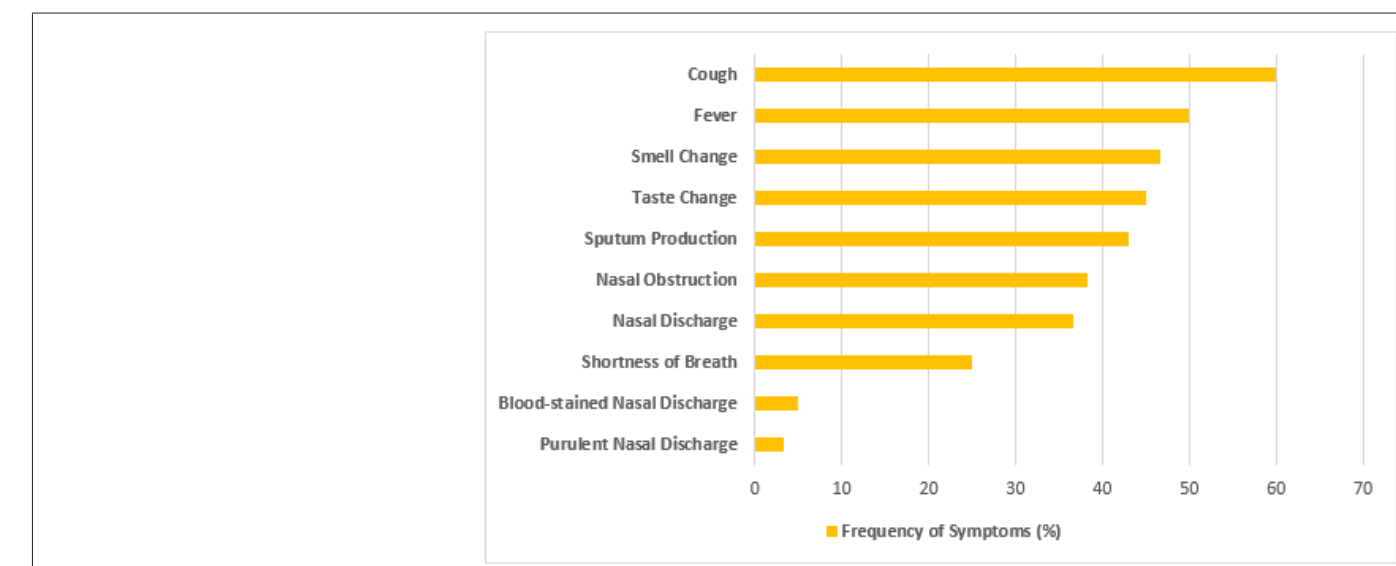

Figure 1: Prevalence of symptoms in patients with confirmed COVID-19. 
In this cross-sectional study, 74 confirmed COVID-19 patients were contacted, and 60 were recruited; 14 declined to participate. Ninety-one control patients were contacted and 60 were recruited; 30 could not be reached by phone and one declined to participate.

\section{Patients' demographic data and presenting symptoms}

The COVID-19 group comprised 36 males and 24 females, while the control group comprised 26 males and 34 females ( $p=0.068$ ). The mean ages were $37.8 \pm 16.6$ years in the COVID-19 group and $38.4 \pm 14.2$ years in the control group $(p=0.818)$. The two groups

Table 1: Demographic data and presenting symptoms of patients. did not differ significantly in terms of basic demographics. The most common symptoms of COVID-19 patients were cough (60\%), fever (50\%) and expectorant (43\%), whereas only 3 to $5 \%$ reported purulent and blood-stained nasal discharge (Figure 1). Dyspnea and fever were more common among confirmed COVID-19 cases than among controls ( $25 \%$ vs $8.3 \%$; OR $3.7, p=0.014$ and $50 \%$ vs $23.3 \%$; OR 3.6; $\mathrm{p}=0.002$, respectively). The presence of rhinorrhea, nasal obstruction, purulent and blood-stained nasal discharge, cough, and sputum production did not differ significantly between the groups (Table 1).

\begin{tabular}{|c|c|c|c|}
\hline Characteristics & COVID-19 Group $(n=60)$ No./total no. (\%) & Control Group $(n=60)$ No./total no. $(\%)$ & p Value \\
\hline Male: Female ratio & $36: 24$ & $26: 34$ & $0.068^{*}$ \\
\hline Age range (years) & $19-71$ & $18-65$ & \\
\hline Mean age (years) & $37.8 \pm 16.6$ & $38.4 \pm 14.2$ & $0.818^{* *}$ \\
\hline History of allergic rhinitis & $13 / 60(21.6)$ & $16 / 60(35)$ & $0.522^{*}$ \\
\hline Medication for nasal symptoms & $2 / 60(3.33)$ & $6 / 60(10)$ & $0.143^{*}$ \\
\hline History of nasal surgery & $0 / 60(0)$ & $0 / 60(0)$ & \\
\hline Rhinorrhea & $22 / 60(36.7)$ & $27 / 60(45)$ & $0.353^{*}$ \\
\hline Nasal blockage & $23 / 60(38.3)$ & $21 / 60(35)$ & $0.705^{*}$ \\
\hline Purulent nasal discharge & $2 / 60(3.33)$ & $1 / 60(1.67)$ & $0.559^{*}$ \\
\hline Epistaxis & $3 / 83(5)$ & $3 / 60(5.0)$ & $1^{*}$ \\
\hline Cough & $36 / 60(60)$ & $28 / 60(46.7)$ & $0.143^{*}$ \\
\hline Expectorant & $26 / 60(43.3)$ & $25 / 60(41.7)$ & $0.853^{*}$ \\
\hline Dyspnea & $15 / 60(25)$ & $5 / 60(8.3)$ & $0.014^{*}$ \\
\hline Subjective fever & $30 / 60(50)$ & $14 / 60(23.3)$ & $0.002^{*}$ \\
\hline Documented fever on admission & $15 / 60(25)$ & $7 / 60(13.2)$ & $0.059^{*}$ \\
\hline \multicolumn{4}{|l|}{ Smell change } \\
\hline 1 (Anosmia) & $9 / 60(15)$ & $0 / 60(0)$ & \\
\hline $2-5$ & $11 / 60(18.3)$ & $0 / 60(0)$ & \\
\hline $6-9$ & $8 / 60(13.3)$ & $0 / 60(0)$ & \\
\hline 10 (Normal) & $32 / 60(53.3)$ & $60 / 60(100)$ & $0.01 \#$ \\
\hline \multicolumn{4}{|l|}{ Taste change } \\
\hline 1 (Ageusia) & $4 / 60(6.7)$ & $0 / 60(0)$ & \\
\hline $2-5$ & $18 / 60(30)$ & $0 / 60(0)$ & \\
\hline $6-9$ & $5 / 60(8.3)$ & $0 / 60(0)$ & \\
\hline 10 (Normal) & $33 / 60(55)$ & $60 / 60(100)$ & $0.01 \#$ \\
\hline
\end{tabular}

*Pearson's Chi-squared test

${ }^{* *}$ Student's t-test

\#Mann-Whitney $U$ test

\section{Smell dysfunction}

Twenty-eight COVID-19 patients (46.7\%) reported smell dysfunction, with nine (15\%) reporting anosmia and 19 (31.7\%) reporting hyposmia. Of these 28 patients, 20 (71.4\%) patients reported a $>50 \%$ reduction in smell. One patient presented with only smell dysfunction without other symptoms. Three patients reported smell loss as the earliest symptoms among others. No control patients reported any smell dysfunction $(\mathrm{p}<0.05$; Figure 2$)$. The mean age of patients who reported smell dysfunction was 31.6 \pm 11.45 years, and there was no sex predilection in this subgroup ( $p=0.342$ ). Nasal obstruction and fever were more commonly reported within the smell dysfunction subgroup $(\mathrm{p}=0.005$ and $\mathrm{p}$ $=0.01$, respectively) . 


\section{Taste dysfunction}

Twenty-seven COVID-19 patients (45\%) reported a taste dysfunction, with four (6.67\%) reporting ageusia and 23 (38.3\%) reporting hypogeusia. Of these 27 patients, $22(81.5 \%)$ reported a $>50 \%$ reduction in taste. No control patients reported any taste dysfunction ( $p<0.05$; Figure 2). The mean age of patients who reported a taste dysfunction was $30.7 \pm 11.62$ years. A significant correlation was observed between a high degree of smell dysfunction and a high degree of taste dysfunction (Spearman's correlation coefficient $=0.813, \mathrm{p}<0.05$ )

\section{Ct values of PCR assays of nasopharyngeal and throat swabs}

The mean Ct value of SARS-CoV-2 PCR tests in the COVID-19 group was $27.4 \pm 6.6$. The Ct values of different subgroups of patients ranged from $26.7 \pm 6.7$ to $34.0 \pm 2.9$ (Table 2 ). The result of one-way ANOVA did not show statistically significant relationship between the $\mathrm{Ct}$ value and the incidence of smell or taste dysfunctions ( $\mathrm{p}=$ 0.822 and $p=0.228$, respectively).

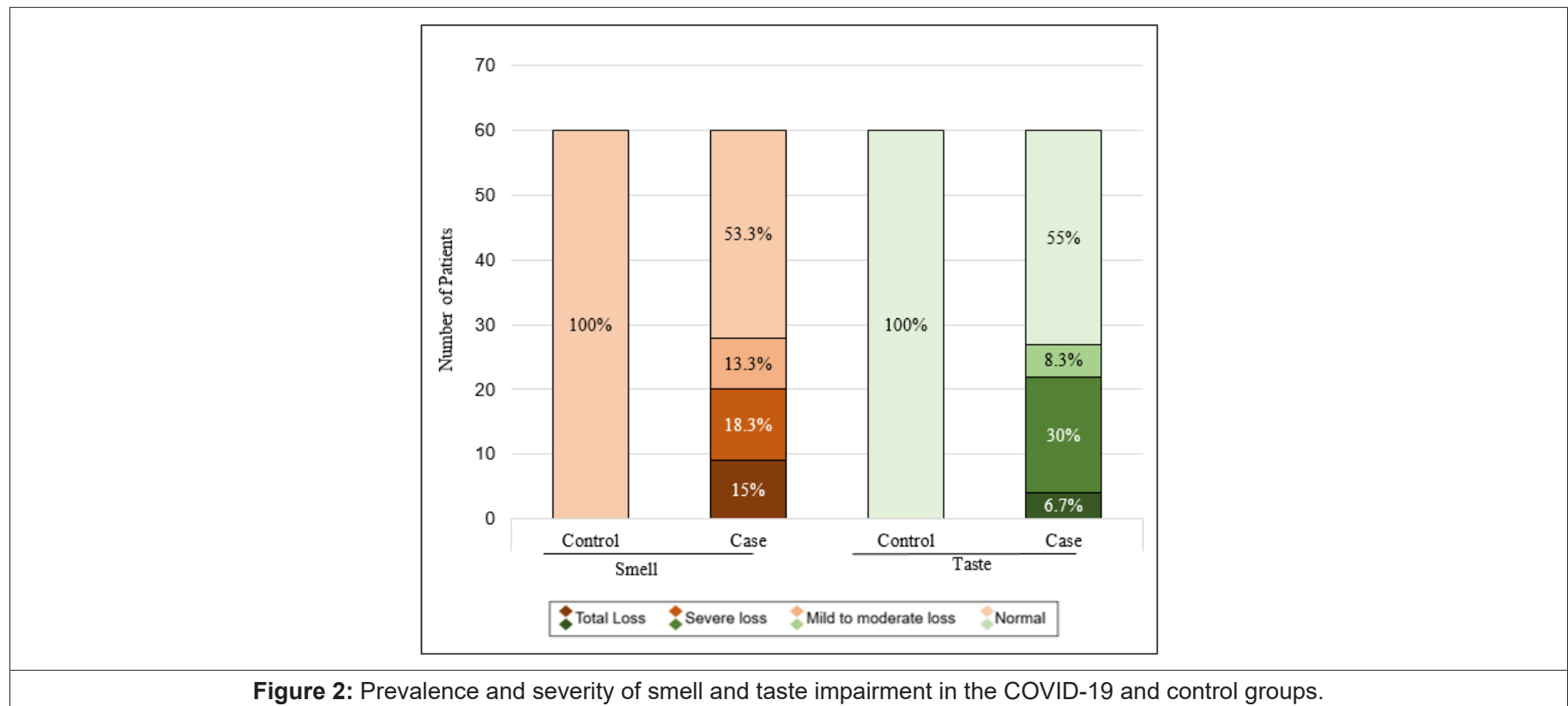

Table 2: Ct values from SARS-CoV-2 PCR assay in patients with confirmed COVID-19.

\begin{tabular}{|c|c|c|c|}
\hline & Number of patients $(n=60)$ & Ct value & p Value \\
\hline \multicolumn{4}{|l|}{ Smell change } \\
\hline 1 (Anosmia) & 9 & $27.5 \pm 6.3$ & \\
\hline $2-5$ & 11 & $29.0 \pm 7.0$ & \\
\hline $6-9$ & 8 & $28.0 \pm 7.2$ & \\
\hline \multirow[t]{2}{*}{10 (Normal) } & 32 & $26.8 \pm 6.7$ & \\
\hline & & & $0.822^{*}$ \\
\hline \multicolumn{4}{|l|}{ Taste change } \\
\hline 1 (Ageusia) & 4 & $34.0 \pm 2.9$ & \\
\hline $2-5$ & 18 & $26.9 \pm 6.0$ & \\
\hline $6-9$ & 5 & $27.8 \pm 6.2$ & \\
\hline \multirow[t]{2}{*}{10 (Normal) } & 33 & $26.8 \pm 7.1$ & \\
\hline & & & $0.228^{*}$ \\
\hline
\end{tabular}

*One-way ANOVA

Ct: cycle threshold; SARS-CoV-2: severe acute respiratory syndrome coronavirus-2; PCR: polymerase chain reaction.

\section{Discussion}

Viral infection is a common cause of acute disturbances in smell and taste, which account for $19 \%$ of all chemosensory disturbances [13]. However, no reports have addressed the incidence of smell and taste dysfunction after viral infections of the upper respiratory tract, possibly because these conditions recover spontaneously or cause only mild symptoms. In our cohort of Chinese patients with COVID-19, the rates of smell and taste dysfunction were $46.7 \%$ and $45 \%$, respectively, which were consistent with symptom reports of 
COVID-19 cases in Western populations (34-88\%) [10,11,14]. The incidence of smell dysfunction was higher in our Chinese patients than in a Korean cohort (15.3\%) [12]. Our findings suggest that there are no ethnic differences in COVID-19-related smell and taste dysfunction between Chinese and Caucasian populations, and the Chinese should take equally high suspicion of infection if sudden smell and taste loss happened during COVID-19 pandemic. Fever and nasal obstruction were more commonly seen in patients suffered from smell impairment which may at least suggest a conductive etiology was present.

In our cohort, no symptomatic patients with negative SARSCoV-2 test results complained of smell or taste impairment whereas Yan et al. also reported a much higher prevalence of smell and taste impairment in COVID-19 patients relative to testnegative patients (68\% vs. $16 \%$ and $71 \%$ vs. $17 \%$, respectively) [11]. This may be attributable to viral influenza season was over in February 2020 and public awareness of self-protection and hygiene required to prevent a viral influenza outbreak. Therefore, the incidence and severity of any non-COVID-19 viral upper respiratory tract infections may cause less disturbance of smell and taste performance. However, this study may have been subject to recall bias. Patients or physicians may also have underrated alterations in patients' ability to smell and taste, due to these being assumptively considered unimportant, short lasting or being overridden by the more sinister symptoms of COVID-19.

It remains unknown why smell and taste impairment was underreported in a very large cohort of confirmed COVID-19 patients in China. A report by the World Health Organization (WHO)-China Joint Mission on COVID-19 that covered the period of February 16-24, 2020 and was published on April 8, 2020 identified fever $(87.9 \%)$, dry cough $(67.7 \%)$, fatigue $(38.1 \%)$, sputum production $(33.4 \%)$ and SOB (18.6\%) as more common symptoms in 55,924 laboratory confirmed COVID-19 cases, but made no mention of taste and smell dysfunction [15]. However, Mao et al. reported smell (5.1\%) and taste disturbances (5.6\%) in 214 patients treated at three designated special care centers in Wuhan, China from January 16 to February 19, 2020 [16]. The incidence of smell and taste dysfunction in the Chinese population of Hong Kong should not be as high in our current report if the difference in symptom presentation between Eastern and Western populations can be attributed to ethnic factors alone. More research is needed to determine whether the differences in symptom presentation could be attributable to different strains of SARS-CoV-2, and this information will become available with the isolation of new coronavirus strains $[17,18]$. Notably, the first report on smell impairment as a marker of COVID-19 was published on March 21, 2020 by the British Rhinologic Society and British Association of Otorhinolaryngology, whereas the WHO-China Joint Mission on COVID-19 and Mao et al. captured data from mid-January to late
February. Most of the subjects recruited in our cohort were tested in March 2020, and most had traveled from the United States, Europe and Japan. More data are needed to determine whether SARS-CoV-2 mutated to a new strain that affects olfactory and gustatory perception.

Hong Kong's reporting criteria for a suspected COVID-19 case are fever, respiratory symptoms or pneumonia and a recent history of travel from any country with active community transmission of COVID-19 or close contact with any confirmed case within 14 days [19]. All suspected cases are tested for SARS-CoV-2, and positive cases are admitted to hospital for surveillance. Although the incidence of fever, SOB, cough, and expectorant ranged from $25 \%$ to $60 \%$ among our confirmed cases, they were also present in 8 to $47 \%$ of control cases. In contrast, smell and taste symptoms were very specific in our series, as we observed frequencies of $46.7 \%$ and $45 \%$, respectively, in the COVID-19 group and no reports in the control group. Our results support the recommendation by the American Academy of Otolaryngology - Head and Neck Surgery advocating the addition of anosmia, hyposmia and dysgeusia to the list of screening tools for possible COVID-19 infection which is also applicable in Chinese population [3].

Real-time PCR yields a Ct value, which is defined as the number of amplification cycles required to reach a threshold of detection of the target nucleic acid. The Ct level is inversely proportional to the amount of the target in a sample. Therefore, the Ct level may indirectly indicate the viral replication activity level, which affects the infectivity of SAR-CoV-2 in a patient [20]. Higher SARS-CoV-2 loads have been detected in the nose than in the throat [21]. In our current study, no correlation was noted between Ct values and smell or taste impairment in individual patients. This suggested that the severity of smell or taste impairment may not be related to the viral activity. Further studies are required to identify the risk factors in causing the smell and taste dysfunction in the COVID-19 patients.

This study is limited by the lack of physical examinations of the nose and pharynx of patients. Additionally, the symptoms related to smell and taste were determined via a questionnaire that inquired about symptoms, and no objective tests were performed to confirm and quantify the severity of these patient-reported abnormalities, and to decide if the smell impairment was conductive or sensorineural in its etiology [22]. Moreover, we could not obtain data from patients who were extremely ill and required intensive care. Those confirmed COVID-19 cases that were discharged may not be medically fit and safe to return physically for further evaluation in otolaryngology clinic due to worry of reactivation of virus for inconsistent PCR results in nasopharyngeal and deep pharyngeal swab. Follow up is only possible by symptoms enquiry through telemedicine until more concrete evidence to rebut the observation of reactivation. 
However, this study is strengthened by its use of a control group with symptoms, which allowed us to demonstrate a much higher prevalence of smell and taste impairment among the COVID-19 cases in Chinese population. Smell impairment could present as the earliest symptom and be the only symptom of some patients. Moreover, assistance from our team allowed us to obtain data from more than two-thirds of the admitted COVID-19 cases at our local facility. While the daily incidence of locally confirmed COVID-19 patients is on single digit recently, it will be lingering on for some time as in some countries like China, Japan, Singapore and Korea. Our real threat is from those asymptomatic carriers who may present lately; the imported cases from countries which are still heavily affected by this pandemic; and another potential seasonal outbreak in fall or winter. Therefore, we still need to be highly vigilant at all time about COVID-19 infection even when global situation seems to ease out in recent weeks.

\section{Conclusion}

We observed no ethnic differences in COVID-19-related smell and taste dysfunction between Chinese and Caucasians. These symptoms were more specific for COVID-19 than fever, cough, and shortness of breath. In this study, smell and taste dysfunction are sufficiently distinctive to be used for screening high-risk subjects for laboratory testing.

\section{Funding}

No funding was received for work described in or related to this study.

\section{Competing and Financial interests}

The authors declare that they have no competing interests and financial relationships to disclose.

\section{Acknowledgements}

The authors would like to thank Monica Lee, MY Chan, BY Ng, J San, SW Chau, HK Wong, SW Chan, SM Ho, IOL Chan, K Wong, CC law, WH Cheung, FC Lee, WL Lo and the infection control teams at United Christian Hospital and Tseung Kwan O Hospital for their efforts regarding the logistical arrangements for this study. We would also like to thank Ms. Agatha Cheng for providing support with data entry and acquisition.

\section{Reference}

1. World Health Organization (2020) Coronavirus disease 2019 (COVID): situation report 119.

2. ENT UK (2020) Loss of sense of smell as marker of COVID-19 infection.

3. (2020) AAO-HNS: Anosmia, hyposmia, and dysgeusia symptoms of coronavirus disease.
4. ENT UK (2020) Advice for patients with new-onset anosmia during COVID-19 pandemic.

5. Vaira LA, Deiana G, Fois AG (2020) Objective evaluation of anosmia and ageusia in COVID-19 patients: Single-center experience on 72 cases. Head Neck.

6. Hopkins C, Surda P, Whitehead E, Kumar BN (2020) Early recovery following new onset anosmia during the COVID-19 pandemic - an observational cohort study. J Otolaryngol Head Neck Surg 49(1): 26.

7. Sun P, Qie S, Liu Z (2020) Clinical characteristics of hospitalized patients with AS-CoV-2 infection: A single arm meta-analysis. J Med Virol PMID: 32108351.

8. Guan WJ, Ni ZY, Hu Y (2020) Clinical characteristics of coronavirus disease 2019 in China. N Engl J Med.

9. Chen N, Zhou M, Dong X, Jieming Qu, Fengyun Gong, et al. (2020) Epidemiological and clinical characteristics of 99 cases of 2019 novel coronavirus pneumonia in Wuhan, China: a descriptive study. Lancet 395(10223): 507-513.

10. Lechien JR, Chiesa-Estomba CM, De Siati DR, Mihaela Horoi, Serge D Le Bon, et al. Olfactory and gustatory dysfunctions as a clinical presentation of mild-to-moderate forms of the coronavirus disease (COVID-19): a multicenter European study. Eur Arch Otorhinolaryngol p. 1-11.

11. Yan CH, Faraji F, Prajapati DP, Boone CE, DeConde AS (2020) Association of chemosensory dysfunction and Covid-19 in patients presenting with influenza-like symptoms. Int Forum Allergy Rhinol

12. Hopkins C, Surda P, Kumar N (2020) Presentation of new onset anosmia during the COVID-19 pandemic. Rhinology 58(3): 295-298.

13. Mott AE, Leopold DA (1991) Disorders in taste and smell. Med Clin North Am 75(6):1321-1353.

14. Giacomelli A, Pezzati L, Conti F, Dario Bernacchia, Matteo Siano, et al. Self-reported olfactory and taste disorders in SARS-CoV-2 patients: a cross-sectional study. Clin Infect Dis.

15. Who Health Organization (2020) Report of the WHO-China joint mission on coronavirus disease 2019 (COVD-19).

16. Ling Mao, Mengdie Wang, Shanghai Chen, Quanwei He, Jiang Chang, et al. (2020) Neurological manifestations of hospitalized patients with COVID-19 in Wuhan, China: a retrospective case series study. JAMA Neurol.

17. (2020) Discovery of 2 strains of COVID-19 coronavirus hints at how it evolved. Forbes.

18. (2020) Deadly coronavirus comes in 3 variants, researchers find. South China Morning Post.

19. (2020) Hong Kong Center for Health Protection. Latest overseas situation of COVID-19 and revised reporting criteria for suspected cases of COVID-19.

20. Joynt GM, Wu WK (2020) Understanding COVID-19: What does viral RNA load really mean? Lancet Infect Dis 20(6): 635-636.

21. Zou L, Ruan F, Huang M, (2020) SARS-CoV-2 Viral load in upper respiratory specimens of infected patients. N Engl J Med 382(12):1177-1179.

22. Seiden AM, Duncan HJ (2001) The Diagnosis of a Conductive Olfactory Loss. Laryngoscope 111(1): 9-14. 